\title{
Extended Factitious Force Approach for Control of a Mobile Manipulator Moving on Unknown Terrain
}

\author{
Wojciech Domski ${ }^{1}$ (D) . Alicja Mazur ${ }^{1} \cdot$ Mirela Kaczmarek $^{1}$
}

Received: 25 October 2017 / Accepted: 11 July 2018 / Published online: 24 July 2018

(C) The Author(s) 2018

\begin{abstract}
In the paper a new control algorithm for mobile manipulators with skid-steering platform, moving on unknown terrain, has been introduced. The mobile manipulator consists of a skid-steering mobile platform and a $2 \mathrm{R}$ rigid manipulating arm mounted on the platform. Due to under-actuation of such mobile platforms, the concept of factitious force (which does not exist in reality and is assumed to be always 0 ) has been utilised. Presented control law, in adaptive and robust version, guarantees asymptotically fast convergence of tracking errors to 0 , which has been proved in the article. Simulations, which have been conducted for object of considerations, confirm that the developed control law works properly even in terrain which parameters are unknown and piece-wise constant.
\end{abstract}

Keywords Mobile manipulator · Underactuated system · Nonholonomic constraint · Skid steering · Kinematics $\cdot$ Dynamics $\cdot$ Adaptive algorithm $\cdot$ Robust algorithm

\section{Introduction}

Mobile manipulators are vivid research topic because of the important role in robotics and possible applications in real life. Those vehicles can be treated separately as mobile platform for transportation purposes or as independent robots conducting complex tasks and requiring certain degree of dexterity. For those task mobile manipulators are good example [10]. Thanks to the good manoeuvrability and high power efficiency mobile manipulators increased their popularity in the industry.

In the motion of wheeled mobile manipulators some interesting issues occur:

1. Problem of wheels' motion. Depending on wheels' type and the way in which they are fixed to the chassis,

Wojciech Domski

wojciech.domski@pwr.edu.pl

Alicja Mazur

alicja.mazur@pwr.edu.pl

Mirela Kaczmarek

mirela.kaczmarek@pwr.edu.pl

1 Chair of Cybernetics and Robotics, Faculty of Electronics, Wrocław University of Science and Technology, ul.Janiszewskiego 11/17, 50-372 Wrocław, Poland motion of wheeled mobile platforms can be performed with or without slipping effect [13]. If platform motion is realized based on the lack of slippage phenomenon between wheels and surface, then there exist equations describing forbidden directions for realized velocities of the system. Such equations are called nonholonomic constraints in platform motion.

Skid-steering mobile platforms (SSMPs) are one kind of nonholonomic wheeled mobile platforms which are equipped with two or more fixed axles of fixed wheels. These platforms are called skid-steering mobile platforms due to skidding effect observed during the motion: they can not change orientation without lateral slipping of wheels. Such SSMPs are actuated only by two motors on left and right side of vehicle. The change of the platform's orientation is achieved by evoking differential velocity. It does not mean that such platforms do not have nonholonomic constraints: they define restricted direction of wheels' motion i.e. lack of longitudinal slippage, not lateral one. Depending on what kind of motion features are to be achieved the wheels on each side of the vehicle can be coupled or not. One advantage of coupled wheels is potential increase in power output due to some ground irregularity - if one wheel is not in contact with the surface it can still transmit power to the other wheel via caterpillar track. 
2. Problem of coupled dynamics. Such a combined system as the wheeled mobile manipulator is able to perform manipulation tasks in a much larger workspace than a fixed-base manipulator but this approach introduces a few of new issues that are not present in the each subsystem considered separately:

- The dynamics of the combined system are much more complicated because they include dynamic interactions between mobile platform and manipulator, see e.g. [20]. In other words, if the manipulator moves it can generate the motion of the uncontrolled platform and the same in back direction.

- If the nonholonomic constraints are present in motion of any subsystem, then the whole combined system has to be considered as a nonholonomic system. It is consequence of the fact that nonholonomic constraints change the mathematical model qualitatively.

- Some problems with definition of the desired trajectory for the mobile manipulators occur many tasks can be realized as the motion of only one subsystem, e.g. only platform's motion or only manipulator's motion, or as a coordinated motion of both subsystems. Therefore desired trajectory for the mobile manipulator's usually is not unique.

Designing control algorithms for skid-steering mobile platforms is a challenging task due to structural slippage occurring in platform motion and underactuation of the dynamics.

Structural slippage In [3] singular perturbation approach has been applied to describe and include slipping effects into mathematical model of wheeled mobile robot. Such method was valid only for very small value of slipping variable and, moreover, models obtained for the motion with and without slippage effect were inconsistent. Some attempt to extend method of singular perturbation into larger allowed slippage was presented in [21]. In turn, in [15] the authors have incorporated sliding variable into dynamical model of nonholonomic system. Nevertheless, it is necessary to take into account slipping phenomenon during design of control law for SSMP platform.

Underactuated dynamics The next problem in control of the SSMP platforms is the underactuation of the dynamics. This issue will be explained next in the text. There are two possible solutions to this problem: introducing of additional constraint or additional input. In [2] the first method for underactuation problem has been utilized. The authors have introduced some artificial constraint on lateral slipping but it was problematic because it is stationarity. Different approach to the control problem for skid-steering platform has been presented in [16]. Authors have shown that skidsteering mobile platform is an underactuated system on dynamic level with non-stationary velocity constraint (nonstationary kinematics). The same idea can be found in [14] and [9].

This paper focuses on the second possible approach to solve the under-actuation problem - it is extended factitious force method where the first announcement about this method, for single factitious force, can be found in [11]. The main idea of proposed method was to add some artificial control inputs, so-called "factitious forces", to make input matrix $B^{*}$ square and invertible. That allowed the problem of an under-actuated dynamic system to be solved - a system with less physical inputs then derived from model. Later, work incorporating more than one factitious force was presented in [12] for a mobile platform. The method was also successfully deployed for a mobile manipulator [4]. In this paper a model of the mobile SSMP platform with $2 \mathrm{R}$ manipulator was investigated. However, in this article an adaptive algorithm [6] and robust algorithm are used to improve control quality in unknown terrain in terms of friction forces during the contact of platform wheels with the surface. The article presents results obtained during the simulations for mentioned two control algorithms - adaptive and robust dynamic control algorithm.

The main contribution of this article is to solve underactuation problem of SSMP platform using extended factitious force to control a mobile manipulator in unknown terrain with one of two presented algorithms.

The paper is organized as follows. Section 2 presents theoretical designing of mathematical models of nonholonomic SSMP platform with $2 \mathrm{R}$ manipulator, expressed in different coordinates - generalized coordinates and auxiliary velocities. In Section 3 concept of extended factitious force in control of nonholonomic SSMP platforms is presented. Section 4 contains formulation of control problem considered in this paper. In Section 5 main result, i.e. new control algorithm including kinematic and dynamic control law, is designed. Section 6 contains results of simulation study. Section 7 presents some conclusions and final remarks.

\section{Mathematical Model}

The object of our considerations is so-called skid-steering mobile platform, i.e. a cart equipped in four fixed wheels mounted on two axes, namely two on the front axis and two on the rear axis, as it is presented in Fig. 1.

From definition, fixed wheels can't change orientation relative to the chassis. Most SSMP platforms has only two non-twisting axes but there are some applications with more than two fixed axes, e.g. Argo All Terrain Vehicle by the 


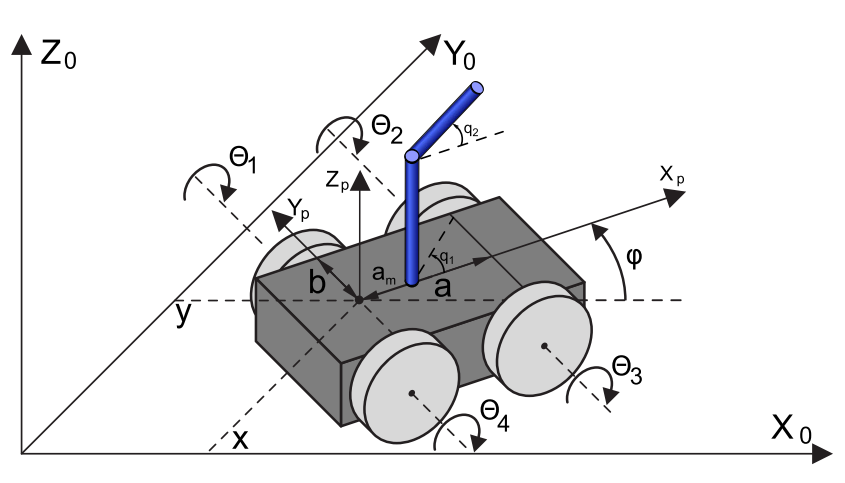

Fig. 1 Object of considerations: SSMP platform with 2R manipulator

Frontline Robotics [1] with three axles. Usually SSMP has only two actuators, one for each side - it is the reason that such kind od the vehicle is often called 'differentially driven robot'. In further considerations it will be assumed that only wheel 1 and 4 (rear axle, see Fig. 1) are actuated.

Geometric parameters of the platform are indicated in Fig. 1: distance between the front and the rear axle is denoted as $a$, symbol $b$ represents half of the platform's width and $a_{m}$ - offset from the rear axle to the middle of the platform. The manipulator's base is located in the mass centre of the platform.

The mobile manipulator is a combined robot consisting of two subsystems: a mobile platform and a rigid manipulator mounted on the top of it. The state vector describing the whole system is equal $q^{T}=\left(q_{m}^{T} q_{r}^{T}\right)$, where $q_{m} \in R^{n}$ and $q_{r} \in R^{p}$. In this paper the manipulator's variables are denoted as $q_{r}=\left(q_{1}, q_{2}\right)^{T}$, while the coordinates of the SSMP are chosen as $q_{m}=$ $\left(x, y, \varphi, \theta_{1}, \theta_{2}, \theta_{3}, \theta_{4}\right)^{T}$. As mentioned before only wheels 1 and 4 are actuated where $\tau_{m 1}$ is a control signal for the first wheel and $\tau_{m 2}$ is a control signal for the fourth wheel.

\subsection{Model in Generalized Coordinates}

The motion of the SSMP platform can be described using generalized coordinates $q_{m} \in R^{n}$ and generalized velocities $\dot{q}_{m} \in R^{n}$. Simultaneously, it will be taken that there is no slippage effect in selected motion directions (no longitudinal slippage) of wheels. It implies the existence of $l$ independent nonholonomic constraints $(l<n)$, which can be presented in the Pfaff form

$A\left(q_{m}\right) \dot{q}_{m}=0$,

where $A$ is a full-rank matrix with dimension $l \times n$,

$A\left(q_{m}\right)=\left[\begin{array}{ccccccc}\cos \varphi & \sin \varphi & -b & -R & 0 & 0 & 0 \\ \cos \varphi & \sin \varphi & b & 0 & 0 & 0 & -R\end{array}\right]$.

The Pfaff matrix describes that there is no longitudinal slippage for the first wheel and for the fourth wheel. Therefore, there are only two constraints in Eq. 2. Since the wheels 1 and 4 are only actuated this is intuitive. If we would allow longitudinal slippage for these wheels then the mobile platform would not move and the wheels would spin.

If the motion of the system is restricted by nonholonomic constraints, then the d'Alembert principle must be evoked to generate the system's dynamics [19]. For the mobile manipulator the dynamics take following form

$$
\begin{aligned}
& {\left[\begin{array}{ll}
Q_{11} & Q_{12} \\
Q_{21} & Q_{22}
\end{array}\right]\left(\begin{array}{c}
\ddot{q}_{m} \\
\ddot{q}_{r}
\end{array}\right)+\left[\begin{array}{cc}
Q_{m} & 0 \\
0 & 0
\end{array}\right]\left(\begin{array}{c}
\ddot{q}_{m} \\
\ddot{q}_{r}
\end{array}\right)+\left(\begin{array}{c}
F \\
0
\end{array}\right)} \\
& +\left[\begin{array}{ll}
C_{11} & C_{12} \\
C_{21} & C_{22}
\end{array}\right]\left(\begin{array}{c}
\dot{q}_{m} \\
\dot{q}_{r}
\end{array}\right)+\left[\begin{array}{cc}
C_{m} & 0 \\
0 & 0
\end{array}\right]\left(\begin{array}{c}
\dot{q}_{m} \\
\dot{q}_{r}
\end{array}\right)+\left(\begin{array}{c}
0 \\
D
\end{array}\right) \\
& =\left[\begin{array}{c}
A^{T} \lambda \\
0
\end{array}\right]+\left[\begin{array}{ll}
B & 0 \\
0 & I
\end{array}\right]\left(\begin{array}{c}
\tau_{m} \\
\tau_{r}
\end{array}\right)
\end{aligned}
$$

where: $Q(q) \in R^{(n+p) \times(n+p)}$ - an inertia matrix for the manipulator with SSMP taken into account, $Q_{m}(q) \in R^{n \times n}$ is an inertia matrix for mobile platform itself, $C(q, \dot{q}) \in$ $R^{(n+p) \times(n+p)}$ - a matrix of Coriolis and centrifugal forces for the whole system, $C_{m}\left(q_{m}, \dot{q}_{m}\right) \in R^{n \times n}$ is a matrix of Coriolis and centrifugal forces for SSMP. In turn, $D \in R^{p}$ is a gravity vector while $F(\dot{q}) \in R^{n}$ is a vector of friction forces between platform's wheels and the terrain, $A\left(q_{m}\right) \in$ $R^{l \times n}-$ a Pfaff matrix defined by Eq. $1, \lambda \in R^{l}-$ a vector of Lagrange multipliers, $B \in R^{(n-l) \times 2}-$ an input matrix for the SSMP, $\tau_{m} \in R^{(n-l)}-$ an input vector of the SSMP, $\tau_{r} \in R^{p}-$ an input vector for the manipulator.

Model (1)-(3) will be called the model of mobile manipulator with SSMP platform expressed in the generalized coordinates.

\subsection{Model in Auxiliary Velocities}

From Eq. 1 we can conclude that velocities of the SSMP platform are always in the kernel of the matrix $A$ i.e. $\dot{q}_{m} \in$ $\operatorname{Ker} A$. It means that the vector $\dot{q}_{m}$ can be represented as a linear combination of some other vectors $g_{i}(q)$ defining a base of the subspace $\operatorname{Ker} A$, with coefficients $\eta_{i}$. Dimension of this subspace equals to $m=n-l$, therefore we can write

$\dot{q}_{m}=\sum_{i=1}^{m} g_{i}(q) \eta_{i}=G\left(q_{m}\right) \eta$,

where $G$ is a full-rank matrix with dimensions $n \times m$ meeting $A G \equiv 0$ relation. To transform the dynamic model expressed in generalized coordinates into the auxiliary velocities it is necessary to calculate

$\ddot{q}_{m}=G\left(q_{m}\right) \dot{\eta}+\dot{G}\left(q_{m}\right) \eta$.

Lagrange multipliers $\lambda$ have to be eliminated - it can be done by the left-side multiplication of platform's equations 
in Eq. 3 by $G^{T}$. In such the case the mathematical model of the nonholonomic mobile manipulator can be rewritten in the following form:

$Q^{*}\left(\begin{array}{c}\dot{\eta} \\ \ddot{q}_{r}\end{array}\right)+C^{*}\left(\begin{array}{c}\eta \\ \dot{q}_{r}\end{array}\right)+D^{*}+F^{*}=B^{*}\left(\begin{array}{c}\tau_{m} \\ \tau_{r}\end{array}\right)$,

where

$$
\begin{aligned}
Q^{*} & =\left[\begin{array}{cc}
G^{T}\left(Q_{11}+Q_{m}\right) G & G^{T} Q_{12} \\
Q_{21} G & Q_{22}
\end{array}\right] \\
C^{*} & =\left[\begin{array}{cc}
G^{T}\left(C_{11}+C_{m}\right) G+G^{T}\left(Q_{11}+Q_{m}\right) \dot{G} & G^{T} C_{12} \\
Q_{21} \dot{G}+C_{21} G & C_{22}
\end{array}\right] \\
D^{*} & =\left(\begin{array}{c}
0 \\
D
\end{array}\right) \quad F^{*}=\left(\begin{array}{c}
G^{T} F \\
0
\end{array}\right) \\
B^{*} & =\left[\begin{array}{cc}
G^{T} B & 0 \\
0 & I
\end{array}\right]=\left[\begin{array}{cc}
B_{m}^{*} & 0 \\
0 & I
\end{array}\right]
\end{aligned}
$$

The model (5) has some structural property which plays important role from the control point of view.

Property 1 [5] For a mobile manipulator with a wheeled nonholonomic mobile platform a skew-symmetry between inertia matrix $Q^{*}$ and the matrix of Coriolis and centrifugal forces $C^{*}$ does not hold anymore. To regain the skewsymmetry, a special correction matrix $C_{K}$ should be added

$$
\frac{d}{d t} Q^{*}=\left(C^{*}+C_{K}\right)+\left(C^{*}+C_{K}\right)^{T} .
$$

The correction matrix $C_{K}$ should be calculated before regulation process. It can be selected for example as a symmetric matrix due to formula given below

$C_{K}=\frac{1}{2}\left\{Q^{*}-C^{*}-\left(C^{*}\right)^{T}\right\}$.

The model (5) is necessary for the designing the control algorithm for the mobile manipulator. It is worth to be emphasised that the mathematical model of the object consists of two parts - the dynamic model (5) and equations of kinematic nonholonomic constraints (4).

\section{Idea of Extended Factitious Force}

Mobile platform can be modelled as a nonholonomic system, especially if it moves without slippage of wheels. Such system can be described by two groups of equations: kinematics (i.e. constraints' equations) and dynamics.

The kinematics of the wheeled mobile platform can be expressed as well-known driftless control system

$\dot{q}=G(q) \eta$,

where auxiliary velocities $\eta \in R^{n-l}$ play role of "controls". They are not the real control inputs because they can not be commanded directly $-\eta$ represent a vector of embedded control inputs, which ensure realization of the task for the kinematics (nonholonomic constraints) if the dynamics were not present. Number of "control inputs" $\eta$ equals $m=$ $n-l$, where $n$ is a dimension of state variables and $l$ is a number of independent nonholonomic constraints. It comes from the fact that any nonholonomic constraint imposed on robot's motion decreases number of controls in driftless control system by one [13]. The number $m$ will be called "number of admissible control inputs" as a consequence of theoretical restrictions.

Next step, after calculation of the nonholonomic constraints, is to obtain the dynamics of the object. Usually, the dynamics of mobile platform have the same number of physical inputs $\tau_{m}$ as a number of auxiliary velocities $\eta$.

\subsection{Under-Actuation of SSMP Platform}

However, in rare cases, nonholonomic robotic systems have lower number of inputs than admissible number $m$. Such situation occurs e.g. in differentially driven mobile platforms (skid-steering platforms, tanks etc.), where there are only two control inputs $\tau_{m}$, each for all wheels for one side of the vehicle. In turn, the most important part of the dynamics (5) is the input matrix to the platform, i.e. $G^{T} B=B_{m}^{*}$, see Eq. 6. Its dimension is

$B_{m}^{*} \in R^{m \times 2}$,

where $m=n-l$. Because the number of actuators used in mechanical construction is smaller than the number of admissible (theoretically applicable) control inputs

$\tau_{m} \in R^{2} \quad 2<m$,

therefore the system is under-actuated on the dynamic level. It means that $B_{m}^{*}$ is rectangular matrix, thus it can not be directly inverted. It is a challenging problem from the control theory point of view because for the dynamics a classical dynamic control algorithms can not be used.

There are two ways to solve the problem of inverting $B_{m}^{*}$ matrix - the matrix standing before physical controls $\tau_{m}$ :

- avoid problem of missing controls assuming additional artificial constraints. Then model in auxiliary velocities has the same size of reduced state variables as the number of control inputs.

- avoid problem of missing control inputs assuming additional factitious inputs. Then model in auxiliary velocities has the same number of state variables and control inputs.

As it has been mentioned in the introduction, the first approach was explored intensively in recent years. The second approach uses so-called "factitious forces" method to make the input matrix square and invertible. 


\subsection{Factitious Force - What is This?}

Factitious forces are the artificial input signals, which are formally attached to the system, making input matrix $B_{m}^{*}$ square. From theoretical point of view they are present (input matrix is square) but they don't exist in reality, therefore it is necessary to set signals coming to these factitious inputs to zero, see Eq. 8 for explanation.

The factitious force method can be used when a model of a vehicle is expressed in auxiliary velocities. One of the important control problems with dynamically underactuated mobile platforms is rectangular input matrix $B_{m}^{*}$. One way of obtaining a square and invertible input matrix is to extend it with additional columns which represent artificial inputs. This can be done in two steps:

1. Extend control forces acting on object with additional forces that are equal to 0 , as follows, e.g. for three factitious forces it can be done as below

$$
B_{m}^{*} \tau_{m}=\left[\begin{array}{cc}
\frac{2}{r} & 0 \\
0 & \frac{2}{r} \\
0 & 0 \\
0 & 0 \\
0 & 0
\end{array}\right]\left(\begin{array}{l}
\tau_{m 1} \\
\tau_{m 2}
\end{array}\right)=\left[\begin{array}{cc|ccc}
\frac{2}{r} & 0 & 0 & 0 & 0 \\
0 & \frac{2}{r} & 0 & 0 & 0 \\
0 & 0 & 1 & 0 & 0 \\
0 & 0 & 0 & 1 & 0 \\
0 & 0 & 0 & 0 & 1
\end{array}\right]\left(\begin{array}{c}
\tau_{m 1} \\
\tau_{m 2} \\
\tau_{m 3 v} \\
\tau_{m 4 v} \\
\tau_{m 5 v}
\end{array}\right)
$$$$
=\left[\begin{array}{cc|ccc}
\frac{2}{r} & 0 & 0 & 0 & 0 \\
0 & \frac{2}{r} & 0 & 0 & 0 \\
0 & 0 & 1 & 0 & 0 \\
0 & 0 & 0 & 1 & 0 \\
0 & 0 & 0 & 0 & 1
\end{array}\right]\left(\begin{array}{c}
\tau_{m 1} \\
\tau_{m 2} \\
0 \\
0 \\
0
\end{array}\right)=B_{e}^{*} \tau_{m e}
$$

2. Extend vector of control signals $\tau_{m}$ with virtual inputs $\tau_{m v}$ which are equal to 0 , therefore all elements from additional columns will have no influence on the model. It means that in the Eq. 8 virtual input signals are equal to 0 , i.e.

$$
\tau_{m 3 v} \equiv 0 \quad \tau_{m 4 v} \equiv 0 \quad \tau_{m 5 v} \equiv 0 .
$$

Moreover, equations describing factitious forces (9) are in fact implicit functions, from which some additional variables could be extracted, in particular:

$$
\begin{aligned}
& \tau_{m 3 v}=h_{1}\left(q_{m}, \dot{q}_{m}, q_{r}, \dot{q}_{r}, q_{m d}, \dot{q}_{m d}, \eta_{2}, \eta_{3}, \eta_{3 r}, \dot{\eta}_{3 r}\right) \\
& \tau_{m 4 v}=h_{2}\left(q_{m}, \dot{q}_{m}, q_{m d}, \dot{q}_{m d}, \eta_{4}, \eta_{4 r}, \dot{\eta}_{4 r}\right) \\
& \tau_{m 5 v}=h_{3}\left(q_{m}, \dot{q}_{m}, q_{m d}, \dot{q}_{m d}, \eta_{5}, \eta_{5 r}, \dot{\eta}_{5 r}\right)
\end{aligned}
$$

As it can be seen above, in the factitious force method the equations defining additional inputs can be used as reference trajectory generators for sliding variables. The factitious force method can be further exploited to increase the number of additional control inputs if needed.
Successful research was undertaken in [12] where this method was used for generating few reference trajectories for different nonholonomic constrains.

From above implicit functions (10) - (12) the reference signals $\dot{\eta}_{3 r}, \dot{\eta}_{4 r}$ and $\dot{\eta}_{5 r}$ can be calculated as presented later.

\section{Control Problem Statement}

In this paper a trajectory tracking problem of a mobile manipulator moving on unknown terrain is considered. Further in the text it will be assumed that the desired trajectory of the whole system can be decomposed onto two subtrajectories, defined for any subsystem separately:

- $q_{m d}(t)$ - the desired trajectory of the mobile platform,

- $q_{r d}(t)$ - the desired trajectory of the manipulator.

Both trajectories are considered to be $C^{2}$ class (twice differentiable). The mobile platform should track desired trajectory $q_{m d}$ while not violating partial lack of slippage on selected wheels. Also the onboard manipulator should track desired joint trajectory $q_{r d}$.

We address the following control problems:

1. Define such a control law $\tau_{m}$ that the the platform with fully known dynamics but moving on unknown terrain tracks desired trajectory $q_{m d}(t)$.

2. Define such a control law $\tau_{r}$ that allows the manipulator with fully known dynamics to track desired joint trajectory $q_{r d}(t)$.

3. During the motion all nonholonomic constraints should be fulfilled.

4. All factitious forces should be equal to 0 .

While considering the whole system (4)-(5) the mobile manipulator's equations can be represented as a cascade with two stages. It is necessary to emphasize that cascaded structure of the mathematical equations doesn't come from the fact that mobile manipulator consists of two different mechanical objects (manipulator and platform) but it is consequence of the presence of nonholonomic constraints.

The first stage of cascade is the kinematics, i.e. set of the equations describing nonholonomic constraints (4), and the second stage is the dynamics (5) of the system. Due to cascaded structure of the mathematical model of the system, the control algorithm has to include two controllers working simultaneously for each stage of cascade - it is so-called backstepping-like algorithm [8]. Draft of such system was presented in Fig. 2.

The cascaded control system was divided into two parts:

- kinematic controller - it generates velocity signal $\eta_{r}$ which should be realized to fulfil the task of trajectory tracking by nonholonomic system. It solves 


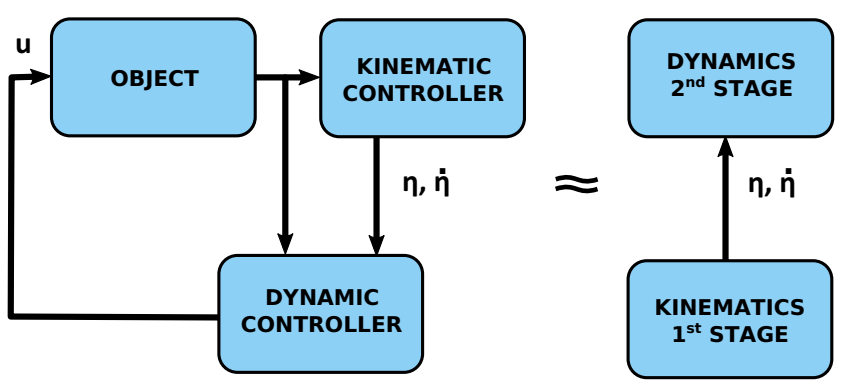

Fig. 2 Backstepping algorithm for a two-staged cascade system

geometrically the problem of going to desired trajectory without wheels' slippage.

- dynamic controller - ensures that the velocity profile $\eta_{r}$ is reconstructed by the system. However, the controller does not influence the platform directly, the $\tau_{m}$ control signals push real velocities $\eta$ towards $\eta_{r}$, previously designed in kinematic control.

\section{Control Algorithm}

This section employs four parts - kinematic controller and dynamic controller in three versions - non-adaptive, adaptive and robust. To ensure proper work of the proposed system two parts (kinematic and one version of dynamic controllers) have to work simultaneously.

\subsection{Kinematic Controller}

The kinematic controller allows the motion planning of the SSMP. It translates trajectory expressed in generalized coordinates into velocity profile $\eta_{r}$ due to the relationship (4), that allows to track desired trajectory while fulfilling nonholonomic constrains

$$
\left(\begin{array}{c}
\dot{x} \\
\dot{y} \\
\dot{\varphi} \\
\dot{\theta}_{1} \\
\dot{\theta}_{2} \\
\dot{\theta}_{3} \\
\dot{\theta}_{4}
\end{array}\right)=\left[\begin{array}{ccccc}
R \cos \varphi & 0 & R \sin \varphi & 0 & 0 \\
R \sin \varphi & 0 & -R \cos \varphi & 0 & 0 \\
0 & R & 0 & 0 & 0 \\
\hline 1 & -b & 0 & 0 & 0 \\
1 & -b & 0 & 1 & 0 \\
1 & b & 0 & 0 & 1 \\
1 & b & 0 & 0 & 0
\end{array}\right]\left(\begin{array}{l}
\eta_{1} \\
\eta_{2} \\
\eta_{3} \\
\eta_{4} \\
\eta_{5}
\end{array}\right)
$$

The wheels angles $\theta_{i}, i=1, . ., 4$ can be obtained after integration of lower part of constraints (15). It means that such constraints are holonomic and they can be removed from vector of state variables.

Similarly to the authors of [15], the upper part of the constraints (13) can be expressed as a combination of velocities calculated for ideal case (motion without slippage) and sliding variables

$\dot{q}=G_{0}(q) \eta+A(q) \mu$

where $\mu$ is a vector of sliding variables. Since the kinematic constraints are not satisfied, $\dot{q}$ does not belong to the space generated by the columns of $G(q)$ but also has a component in the orthogonal complement of this space. Nonholonomic part of (13) can be expressed as

$$
\begin{aligned}
\left(\begin{array}{c}
\dot{x} \\
\dot{y} \\
\dot{\varphi}
\end{array}\right) & =\left[\begin{array}{ccccc}
R \cos \varphi & 0 & R \sin \varphi & 0 & 0 \\
R \sin \varphi & 0 & -R \cos \varphi & 0 & 0 \\
0 & R & 0 & 0 & 0
\end{array}\right]\left(\begin{array}{l}
\eta_{1} \\
\eta_{2} \\
\eta_{3} \\
\eta_{4} \\
\eta_{5}
\end{array}\right) \\
& =\left[\begin{array}{ccc}
R \cos \varphi & 0 \\
R \sin \varphi & 0 \\
0 & R
\end{array}\right]\left(\begin{array}{l}
\eta_{1} \\
\eta_{2}
\end{array}\right)+\left(\begin{array}{c}
R \sin \varphi \\
-R \cos \varphi \\
0
\end{array}\right) \eta_{3} .
\end{aligned}
$$

Restricting our attention only for platform's state variables and assuming slippage-less motion (i.e. $\eta_{3}=\eta_{4}=$ $\eta_{5}=0$ ) we get kinematic (4) for ideal case which is in fact the same as for the unicycle's kinematics

$\left(\begin{array}{c}\dot{x} \\ \dot{y} \\ \dot{\varphi}\end{array}\right)=\left[\begin{array}{cc}\cos \varphi & 0 \\ \sin \varphi & 0 \\ 0 & 1\end{array}\right]\left(\begin{array}{c}v \\ \omega\end{array}\right)$,

where

$v=R \eta_{1}, \quad \omega=R \eta_{2}$.

However, the unicycle's equations do not consider slippage phenomenon which in turn occurs while the SSMP is moving. Therefore approximation of real model was done using equations describing $(2,0)$ class robot (i.e. unicycle). This leads to another velocity profile which resembles an approximation of slippage occurring during the motion. Therefore, it can be used to incorporate additional virtual inputs in extended factitious force method.

In further considerations only admissible desired trajectories will be realized. It means that they fulfil

$\left(\begin{array}{c}\dot{x}_{d} \\ \dot{y}_{d} \\ \dot{\varphi}_{d}\end{array}\right)=\left[\begin{array}{cc}\cos \varphi_{d} & 0 \\ \sin \varphi_{d} & 0 \\ 0 & 1\end{array}\right]\left(\begin{array}{c}v_{d} \\ \omega_{d}\end{array}\right)$.

In turn, reference tracking errors are defined as follows

$\left(\begin{array}{l}x_{e} \\ y_{e} \\ \varphi_{e}\end{array}\right)=\operatorname{Rot}(z,-\varphi)\left(\begin{array}{c}x-x_{d} \\ y-y_{d} \\ \varphi-\varphi_{d}\end{array}\right)$.

It is obvious that convergence to 0 of reference tracking errors $x_{e}, y_{e}, \varphi_{e}$ implies trajectory tracking of the unicycle's platform. 
Reference dynamics errors calculated from Eqs. 16 and 17 can be expressed as below

$$
\begin{aligned}
& \dot{x}_{e}=\omega y_{e}+v-v_{d} \cos \varphi_{e} \\
& \dot{y}_{e}=-\omega x_{e}+v_{d} \sin \varphi_{e} \\
& \dot{\varphi}_{e}=\omega-\omega_{d} .
\end{aligned}
$$

Proof of the convergence of the kinematic controller Let's consider following Lyapunov-like function

$V_{k}\left(x_{e}, y_{e}, \varphi_{e}\right)=\frac{1}{2} x_{e}^{2}+\frac{1}{2} y_{e}^{2}+\frac{1}{2} \varphi_{e}^{2}$,

which is non-negative definite. Time derivative of $V_{k}$ calculated along solutions of the system (18) equals

$$
\begin{aligned}
\dot{V}_{k} & =x_{e} \dot{x}_{e}+y_{e} \dot{y}_{e}+\varphi_{e} \dot{\varphi}_{e} \\
& =x_{e}\left(v-v_{d} \cos \varphi_{e}\right)+\varphi_{e}\left(\omega-\omega_{d}+v_{d} y_{e} \frac{\sin \varphi_{e}}{\varphi_{e}}\right) .
\end{aligned}
$$

Equations describing kinematic controller were presented in [17]

$$
\left(\begin{array}{c}
v_{r} \\
\omega_{r}
\end{array}\right)=\left(\begin{array}{c}
-k_{1} x_{e}+v_{d} \cos \varphi_{e} \\
\omega_{d}-k_{2} \varphi_{e}-v_{d} y_{e} \frac{\sin _{\varphi_{e}}}{\varphi_{e}}
\end{array}\right),
$$

where $k_{1}, k_{2}>0, \eta_{1 d}, \eta_{2 d}$ and $x_{d}, y_{d}, \varphi_{d}$ are desired velocities and positions of the unicycle with desired trajectory $q_{m d}(t)$ and $R$ is a radius of a wheel. Signals

$\eta_{1 r}=\frac{v_{r}}{R}, \quad \eta_{2 r}=\frac{\omega_{r}}{R}$

are reference velocities generated by the kinematic controller and passed to the dynamic controller for trajectory tracking task.

Putting designed kinematic control law (20) into (19) we obtain

$\dot{V}_{k}=-k_{1} x_{e}^{2}-k_{2} \varphi_{e}^{2} \leq 0$.

Now we use the lemma coming from Barbalat to prove asymptotic stability of the kinematic controller (20).

Lemma 1 (Barbalat) Let $f(t)$ be some real $C^{2}$ function. Assume that $f(t)$ is limited from below and $\dot{f}(t) \leq 0$. If $\ddot{f}(t)$ is bounded function $(\ddot{f}(\cdot) \in B)$, then

$\lim _{t \rightarrow+\infty} \dot{f}(t)=0$.

From Eq. 21 we can conclude that $V_{k}$ is decreasing and recently it reaches limit value $V_{\min } \geq 0$. Therefore all error signals $x_{e}, y_{e}, \varphi_{e}$ are bounded. Moreover, all desired trajectories and their first and second derivatives are bounded from assumption. From such reason $\ddot{V}_{k}$ is a multiplication of bounded signals and stays bounded. As a consequence, from Barbalat lemma it implies that

$\lim _{t \rightarrow \infty} x_{e}, \varphi_{e}=0$.
To prove the asymptotic convergence of $y_{e}$ we evoke Barbalat lemma once again. Let's take as a function

$f(t)=\varphi_{e}$.

Such function is bounded from below and its first and second time derivatives are bounded. It means that from Barbalat lemma is can be concluded that

$$
\begin{aligned}
\lim _{t \rightarrow+\infty} \dot{f}(t) & =-k_{2} \varphi_{e}-v_{d} y_{e} \frac{\sin \varphi_{e}}{\varphi_{e}} \\
& =0 .
\end{aligned}
$$

First term on the right side of Eq. 22 goes to zero because $\varphi_{e}$ goes to zero. However, in the second term $v_{d}$ is not equal zero from assumption and $\lim _{t \rightarrow+\infty} \frac{\sin \varphi_{e}}{\varphi_{e}}=1$. It means that only the signal $y_{e}$ must go to zero. It ends the proof of proper action of kinematic controller.

\subsection{Dynamic Controller - Nonadaptive Version}

Dynamic control law is based on the full knowledge about the model so the following control law can be used

$$
\begin{aligned}
\tau= & {\left[\begin{array}{cc}
B_{e}^{*} & 0 \\
0 & I
\end{array}\right]^{-1}\left\{Q^{*}\left(\begin{array}{c}
\dot{\eta}_{r} \\
\ddot{q}_{r e f}
\end{array}\right)+C^{*}\left(\begin{array}{c}
\eta_{r} \\
\dot{q}_{r e f}
\end{array}\right)+D^{*}+F^{*}\right.} \\
& \left.-\left(K_{d}+C_{K}\right)\left(\begin{array}{c}
e_{\eta} \\
s
\end{array}\right)\right\},
\end{aligned}
$$

where $K_{d}=\operatorname{diag}\left\{k_{d}\right\}$ is positive definite diagonal matrix and $C_{K}$ is the correction matrix. It is worthy of note that $F^{*}$ is a vector incorporating unknown friction forces.

All elements of error vectors $e_{\eta}$ and $e_{r}$ are defined as follows

$e_{\eta}=\left(\begin{array}{c}\eta_{1}-\eta_{1 r} \\ \eta_{2}-\eta_{2 r} \\ \eta_{3 v}-\eta_{3 v r} \\ \eta_{4 v}-\eta_{4 v r} \\ \eta_{5 v}-\eta_{5 v r}\end{array}\right), \quad e_{r}=\left(\begin{array}{c}q_{1}-q_{1 d} \\ q_{2}-q_{2 d}\end{array}\right)$,

$s=\dot{q}_{r}-\dot{q}_{r e f}=\dot{e}_{r}+\Lambda e_{r}, \quad \Lambda=\Lambda^{T}>0$.

Signals $\eta=\left(\eta_{1}, \eta_{2}, \eta_{3}, \eta_{4}, \eta_{5}\right)$ can be calculated using nonholonomic constraints (15). Signals $\eta_{3}, \eta_{4}, \eta_{5}$ are the approximations of some slippages occurring in the system. Signals $\eta_{1 r}$ and $\eta_{2 r}$ are generated by the kinematic controller (20) while virtual reference signals $\eta_{v r}$ come from solution to the implicit equations for factitious forces. In considered model there are 3 such signals: $\dot{\eta}_{3 r}, \dot{\eta}_{4 r}$ and $\dot{\eta}_{5 r}$ (26)-(28). Due to complex structure of these signals only symbolic form is presented.

$$
\begin{aligned}
& \dot{\eta}_{3 r}=f_{1}\left(q_{m}, \dot{q}_{m}, q_{r}, \dot{q}_{r}, q_{m d}, \dot{q}_{m d}, \eta_{2}, \eta_{3}, \eta_{3 r}\right), \\
& \dot{\eta}_{4 r}=f_{2}\left(q_{m}, \dot{q}_{m}, q_{m d}, \dot{q}_{m d}, \eta_{4}, \eta_{4 r}\right) \\
& \dot{\eta}_{5 r}=f_{3}\left(q_{m}, \dot{q}_{m}, q_{m d}, \dot{q}_{m d}, \eta_{5}, \eta_{5 r}\right) .
\end{aligned}
$$


Proof of the convergence After substitution control law (23) into the system (5), we get the closed-loop system's dynamics

$Q^{*}\left(\begin{array}{c}\dot{e}_{\eta} \\ \dot{s}\end{array}\right)+\left(C^{*}+C_{K}\right)\left(\begin{array}{c}e_{\eta} \\ s\end{array}\right)+K_{d}\left(\begin{array}{c}e_{\eta} \\ s\end{array}\right)=0$.

For the system (29) we propose following Lyapunov-like function

$V\left(e_{\eta}, s\right)=\frac{1}{2}\left(\begin{array}{ll}e_{\eta}^{T} & s^{T}\end{array}\right) Q^{*}\left(\begin{array}{c}e_{\eta} \\ s\end{array}\right)$,

which is non-negatively defined.

We compute time derivative of $V$ along solutions of the closed-loop system of Eq. 29

$$
\begin{aligned}
\dot{V} & =\frac{1}{2}\left(\begin{array}{ll}
e_{\eta}^{T} & s^{T}
\end{array}\right) \dot{Q}^{*}\left(\begin{array}{c}
e_{\eta} \\
s
\end{array}\right)+\left(\begin{array}{ll}
e_{\eta}^{T} & s^{T}
\end{array}\right) Q^{*}\left(\begin{array}{c}
\dot{e}_{\eta} \\
\dot{s}
\end{array}\right) \\
& =-\left(\begin{array}{ll}
e_{\eta}^{T} & s^{T}
\end{array}\right) K_{d}\left(\begin{array}{c}
e_{\eta} \\
s
\end{array}\right) \leq 0 .
\end{aligned}
$$

Now we evoke the theorem given by La Salle and Yoshizawa [8] to prove a convergence of the dynamic controller.

Theorem 1 (La Salle-Yoshizawa) Let $x=0$ be an equilibrium point of the system

$\dot{x}=f(x, t)$

and suppose $f$ is locally Lipschitz in $x$ uniformly in t. Let $V: R^{n} \times R_{+} \longrightarrow R_{+}$be continuously differentiable function such that

$\gamma_{1}(\|x\|) \leq V(x, t) \leq \gamma_{2}(\|x\|)$,

$\dot{V}=\frac{\partial V}{\partial t}+\frac{\partial V}{\partial x} f(x, t) \leq-W(x) \leq 0$,

$\forall t \geq 0, \forall x \in R^{n}$, where $\gamma_{1}, \gamma_{2}$ are class $K_{\infty}$ functions and $W$ is continuous function. Then, all solutions of Eq. 32 are globally uniformly bounded and satisfy

$\lim _{t \rightarrow+\infty} W(x(t))=0$.

In addition, if $W(x)$ is positive definite, then the equilibrium $x=0$ is globally uniformly asymptotically stable.

From La Salle \& Yoshizawa theorem it could be concluded that the error $e_{\eta}$ converges asymptotically to zero. In turn, convergence of $e_{\eta}$ to zero means that the velocity profile generated by kinematic controller is successfully followed, and therefore one can conclude that the nonholonomic system i.e. skid-steering platform tracks the desired trajectory $q_{m d}$. Moreover, $s$ converges to 0 . From definition (25) of $s$ it can be concluded that $e_{r} \rightarrow 0$ also. It ends the proof.

\subsection{Dynamic Controller - Adaptive Version}

It was assumed that the properties of terrain, on which the mobile manipulator moves, are unknown. To describe this kind of uncertainty, a model of friction was introduced in the following form [2]

$$
F=\left(\begin{array}{c}
0 \\
0 \\
\frac{1}{2} b f_{r} g m_{c}\left(\operatorname{sgn}\left(\dot{q}_{1}+b \dot{q}_{3}\right)-\operatorname{sgn}\left(\dot{q}_{1}-b \dot{q}_{3}\right)\right) \\
\frac{1}{2} g m_{c} \mu \operatorname{sgn}\left(\dot{q}_{2}\right) \\
0 \\
0 \\
\frac{1}{2} g m_{c} \mu \operatorname{sgn}\left(\dot{q}_{2}\right)
\end{array}\right)
$$

where $\operatorname{sgn}(x) \approx \tanh \left(s_{c} x\right)$ and $s_{c}$ defines how closely $\operatorname{sgn}(x)$ function is approximated [7]. The $s_{c}$ term describes how closely the approximation resembles the $\operatorname{sgn}(x)$ function in accordance with

$\lim _{x \rightarrow+\infty} \tanh \left(s_{c} x\right)=\operatorname{sgn}(x)$.

The longitudinal resistive force coefficient $f_{r}$ and lateral friction coefficient $\mu$ are not known. They form following vector of unknown real parameters

$a=\left(\begin{array}{c}f_{r} \\ \mu\end{array}\right)$.

Friction forces can be expressed in the following form

$F^{*}=Y(\dot{q}) a$,

where $Y(\dot{q})$ is so-called regression matrix. Now it is necessary to introduce adaptive control algorithm. Such control algorithm consists of two laws: control law defining input signals to the system and adaptation law, which is a differential equation describing evolution of estimated parameters. Therefore adaptive version of control law will be firstly introduced.

Control law Let's consider control law given below

$$
\begin{aligned}
\tau= & B_{e}^{-1}\left\{Q^{*}\left(\begin{array}{c}
\dot{\eta}_{r} \\
\ddot{q}_{r e f}
\end{array}\right)+C^{*}\left(\begin{array}{c}
\eta_{r} \\
\dot{q}_{r e f}
\end{array}\right)+D^{*}+\hat{F}^{*}\right. \\
& \left.-\left(K_{d}+C_{K}\right)\left(\begin{array}{c}
e_{\eta} \\
s
\end{array}\right)\right\} .
\end{aligned}
$$

The Eq. 38 can be rewritten as follows

$$
\begin{aligned}
\tau= & B_{e}^{-1}\left\{Q^{*}\left(\begin{array}{c}
\dot{\eta}_{r} \\
\ddot{q}_{r e f}
\end{array}\right)+C^{*}\left(\begin{array}{c}
\eta_{r} \\
\dot{q}_{r e f}
\end{array}\right)+D^{*}\right. \\
& \left.+Y(\dot{q}) \hat{a}(t)-\left(K_{d}+C_{K}\right)\left(\begin{array}{c}
e_{\eta} \\
s
\end{array}\right)\right\}
\end{aligned}
$$

where $\hat{a}(t)$ is vector of estimated parameters. 
Adaptation law Differential equation defining a way to change values of estimated parameters is given as [18]

$\dot{\hat{a}}=-\Gamma Y^{T}(\dot{q})\left(\begin{array}{c}e_{\eta} \\ s\end{array}\right)$,

where $\Gamma=\Gamma^{T}>0$ is a matrix of adaptation gains and $Y$ was derived from $F=Y a$.

Proof of the convergence After substitution control law (39) into the system (5), we get the closed-loop system's dynamics as follows

$Q^{*}\left(\begin{array}{c}\dot{e}_{\eta} \\ \dot{s}\end{array}\right)+\left(C^{*}+C_{K}+K_{d}\right)\left(\begin{array}{c}e_{\eta} \\ s\end{array}\right)=Y(\dot{q}) \tilde{a}(t)$,

where $\tilde{a}(t)=\hat{a}(t)-a$ is a vector of the parameter errors. For the system (41) we propose following Lyapunov-like function

$V=\frac{1}{2}\left(e_{\eta}^{T} s^{T}\right) Q^{*}\left(\begin{array}{c}e_{\eta} \\ s\end{array}\right)+\frac{1}{2} \tilde{a}^{T} \Gamma^{-1} \tilde{a} \geq 0$,

which is non-negatively defined. We compute time derivative of $V$ along solutions of the closed-loop system of Eqs. 39, 40

$$
\begin{aligned}
\dot{V}= & \frac{1}{2}\left(\begin{array}{ll}
e_{\eta}^{T} & s^{T}
\end{array}\right) \dot{Q}^{*}\left(\begin{array}{c}
e_{\eta} \\
s
\end{array}\right)+\left(\begin{array}{ll}
e_{\eta}^{T} & s^{T}
\end{array}\right) Q^{*}\left(\begin{array}{c}
\dot{e}_{\eta} \\
\dot{s}
\end{array}\right) \\
& +\tilde{a}^{T} \Gamma^{-1} \dot{\tilde{a}} \\
= & -\left(\begin{array}{ll}
e_{\eta}^{T} & s^{T}
\end{array}\right) K_{d}\left(\begin{array}{c}
e_{\eta} \\
s
\end{array}\right) \leq 0 .
\end{aligned}
$$

From La Salle \& Yoshizawa theorem it could be concluded that the error $e_{\eta}$ converges asymptotically to zero. In turn, convergence of $e_{\eta}$ to zero means that the velocity profile generated by kinematic controller is successfully followed, and therefore one can conclude that the nonholonomic system i.e. skid-steering platform tracks the desired trajectory $q_{m d}$. On the other hand, if time derivative of $V$ is non-positive it means that $V$ is decreasing with time and consequently it tends to some finite value. This ends the proof.

\subsection{Dynamic Controller - Robust Version}

Robust controller can be applied if properties of terrain, on which the mobile manipulator moves, are unknown. However, the dynamics of the object is known.

\section{Assumption 1 Unknown friction coefficients of the ground are in some interval}

$a \in\left[a_{\min }, a_{\max }\right]$

where $a_{\min }$ and $a_{\max }$ are constant and known.
To describe this kind of uncertainty, an approximation of friction forces has a form

$\hat{F}^{*}=Y(\dot{q}) \hat{a}$

where $\hat{a}$ is constant vector of parameters which fulfils Assumption 1, i.e. from the interval given by Eq. 43.

Control law Let's consider dynamic control law for unknown parameters of terrain

$$
\begin{aligned}
\tau= & B_{e}^{-1}\left\{Q^{*}\left(\begin{array}{c}
\dot{\eta}_{r} \\
\ddot{q}_{r e f}
\end{array}\right)+C^{*}\left(\begin{array}{c}
\eta_{r} \\
\dot{q}_{r e f}
\end{array}\right)+D^{*}+\hat{F}^{*}\right. \\
& \left.-\left(K_{d}+C_{K}\right)\left(\begin{array}{c}
e_{\eta} \\
s
\end{array}\right)-K \operatorname{sgn}\left(\begin{array}{c}
e_{\eta} \\
s
\end{array}\right)\right\} .
\end{aligned}
$$

The Eq. 45 can be rewritten in the following way

$$
\begin{aligned}
\tau= & B_{e}^{-1}\left\{Q^{*}\left(\begin{array}{c}
\dot{\eta}_{r} \\
\ddot{q}_{r e f}
\end{array}\right)+C^{*}\left(\begin{array}{c}
\eta_{r} \\
\dot{q}_{r e f}
\end{array}\right)+D^{*}+Y(\dot{q}) \hat{a}\right. \\
& \left.-\left(K_{d}+C_{K}\right)\left(\begin{array}{c}
e_{\eta} \\
s
\end{array}\right)-K \operatorname{sgn}\left(\begin{array}{c}
e_{\eta} \\
s
\end{array}\right)\right\}
\end{aligned}
$$

where $\hat{a}$ is vector of estimated parameters.

Proof of the convergence After substitution control law (46) into the system (5), we get the closed-loop system's dynamics as follows

$$
Q^{*} \dot{e}_{\zeta}+\left(C^{*}+C_{K}+K_{d}\right) e_{\zeta}+K \operatorname{sgn} e_{\zeta}=Y(\dot{q}) \tilde{a},
$$

where symbol $e_{\zeta}$ is defined below

$e_{\zeta}=\left(\begin{array}{c}e_{\eta} \\ s\end{array}\right)$.

For the system (47) we propose following Lyapunov-like function

$V=\frac{1}{2} e_{\zeta}^{T} Q^{*} e_{\zeta} \geq 0$

which is non-negatively defined. We compute time derivative of $V$ along solutions of the closed-loop system (47)

$$
\begin{aligned}
\dot{V} & =\frac{1}{2} e_{\zeta}^{T} \dot{Q}^{*} e_{\zeta}+e_{\zeta}^{T} Q^{*} \dot{e}_{\zeta} \\
& =-e_{\zeta}^{T} K_{d} e_{\zeta}-e_{\zeta}^{T}\left(K s g n e_{\zeta}-Y \tilde{a}\right)
\end{aligned}
$$

In the next step the time derivative of $V$ will be rewritten as below

$$
\begin{aligned}
\dot{V} & =-e_{\zeta}^{T} K_{d} e_{\zeta}-e_{\zeta}^{T}\left(K s g n e_{\zeta}-Y \tilde{a}\right) \\
& =-e_{\zeta}^{T} K_{d} e_{\zeta}-\sum_{i=1}^{n} e_{i \zeta}\left(K s g n e_{i \zeta}-\sum_{j=1}^{p} Y_{i j} \tilde{a}_{j}\right), \\
& =-e_{\zeta}^{T} K_{d} e_{\zeta}-\sum_{i=1}^{n} e_{i \zeta} \operatorname{sgn} e_{i \zeta}\left(K-\operatorname{sgn} e_{i \zeta} \sum_{j=1}^{p} Y_{i j} \tilde{a}_{j}\right), \\
& =-e_{\zeta}^{T} K_{d} e_{\zeta}-\sum_{i=1}^{n}\left|e_{i \zeta}\right|\left(K-\operatorname{sgn} e_{i \zeta} \sum_{j=1}^{p} Y_{i j} \tilde{a}_{j}\right),
\end{aligned}
$$


where $p=2$ is a number of unknown parameters in the dynamics and $\tilde{a}_{j}$ is $j$ th element in vector of parameter errors.

In further evaluations of $\dot{V}$ it will be assumed, that real velocity $\dot{q}_{m}$ of the mobile platform is bounded. It it obvious from practical point of view, but is necessary to complete the proof of the proper working of control algorithm.

Then, if

$K>\sum_{j=1}^{p} Y_{i j} \tilde{a}_{j}+\varepsilon, \quad \varepsilon>0, \quad \forall i=1, \ldots, n$

we get

$\dot{V} \leq-e_{\zeta}^{T} K_{d} e_{\zeta}-\sum_{i=1}^{n}\left|e_{i \zeta}\right| \varepsilon$

Once again, from La Salle \& Yoshizawa theorem, it could be concluded that each element of error $e_{\zeta}$ converges asymptotically to zero. In turn, convergence of $e_{\eta}$ to zero means that the the velocity profile generated by kinematic controller is successfully followed, and therefore one can conclude that the nonholonomic system i.e. skid-steering platform tracks the desired trajectory $q_{m d}$. On the other hand, if

$s=\dot{e}_{r}+\Lambda e_{r} \quad \longrightarrow 0$,

then it implies $e_{r} \rightarrow 0$. This ends the proof.

\section{Simulation Study}

Four scenarios were conducted. For each version of applied algorithm we have prepared two cases, namely tracking of admissible trajectory, a circle, and inadmissible trajectory, a square.

For each set of simulations initial conditions are noted and accompanying set of figures with results was presented. Slippage between the terrain and the robot's wheels was occurring and the parameters of the friction, $\mu$ and $f_{r}$, are constant and unknown to the robot's controller, thus an adaptive law was incorporated (40). Furthermore, friction coefficients are described with time dependant function for each scenario and are as follows:

$f_{r}(t)=\left\{\begin{array}{l}0.10 \text { if } t \leq 50 \\ 0.15 \text { if } t>50 .\end{array}\right.$

$\mu(t)=\left\{\begin{array}{l}0.9 \text { if } t \leq 50 \\ 1.1 \text { if } t>50\end{array}\right.$

The first trajectory (the admissible trajectory) was a circle with radius of $10 \mathrm{~m}$. The vehicle should move with angular velocity equal to $0.1\left[\frac{\mathrm{rad}}{\mathrm{s}}\right]$. The second trajectory, the inadmissible, was a square and the mobile platform should move along its edges with constant linear velocity equal to $0.5\left[\frac{\mathrm{m}}{\mathrm{s}}\right]$.

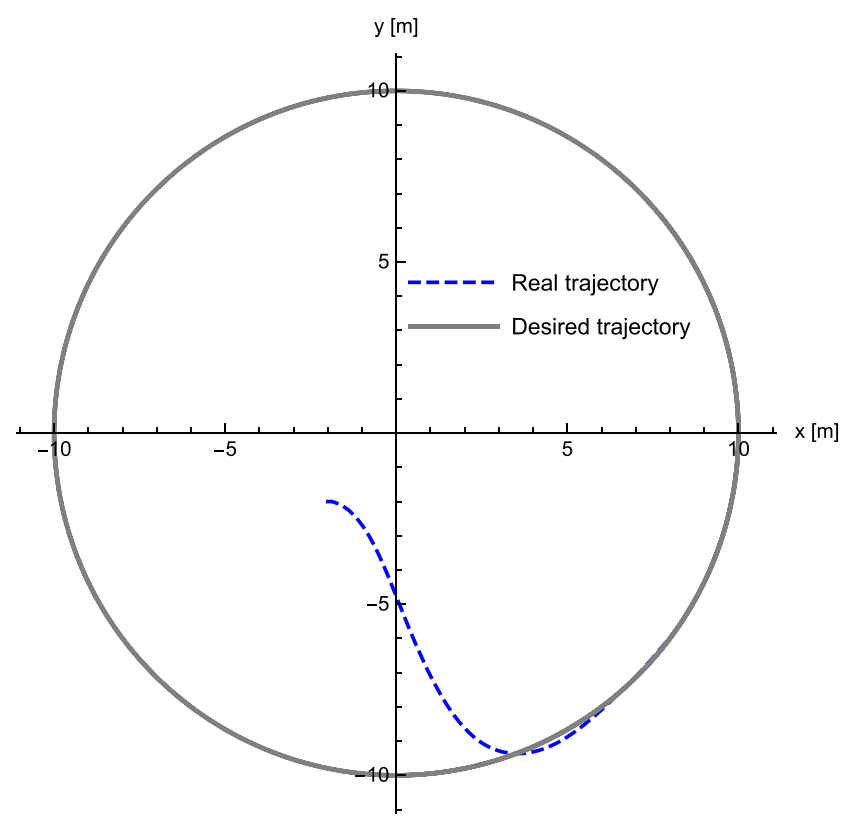

Fig. 3 Real and desired trajectory of the mobile platform

The desired trajectory for $2 \mathrm{R}$ manipulator joints was defined for each joint with following equations:

$q_{1 d}=0.15 \sin (0.150 \pi t)+0.50$,

$q_{2 d}=-0.15 \cos (0.075 \pi t)-0.25$.

Moreover, regulation parameters for kinematic controller were chosen as follows:

$k_{1}=1, \quad k_{2}=1$.

Parameters of the dynamic controller in regard to variables $\Lambda$ and $\Gamma$ were set to:

$\Lambda=\operatorname{diag}\{10\}, \quad \Gamma=\operatorname{diag}\{1\}$.

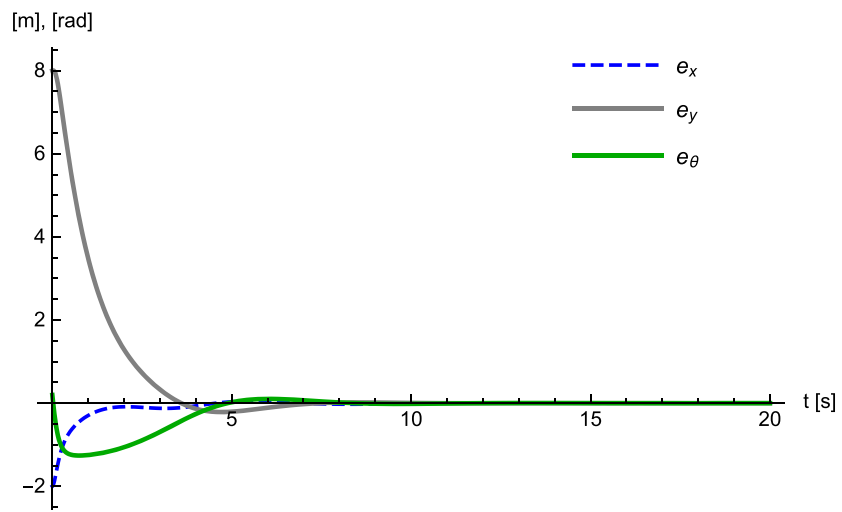

Fig. 4 Errors in Cartesian space - position and orientation of the mobile platform 


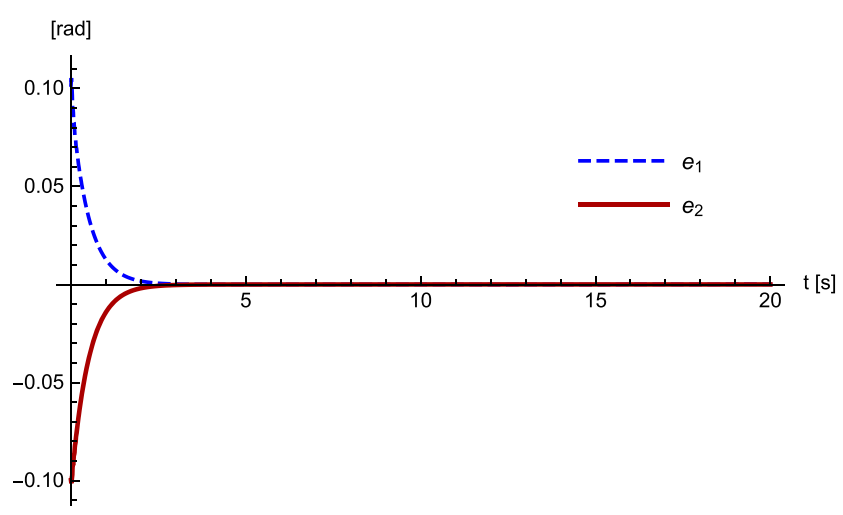

Fig. 5 Error in the manipulator's joint $q_{r 1}$ and $q_{r 2}$

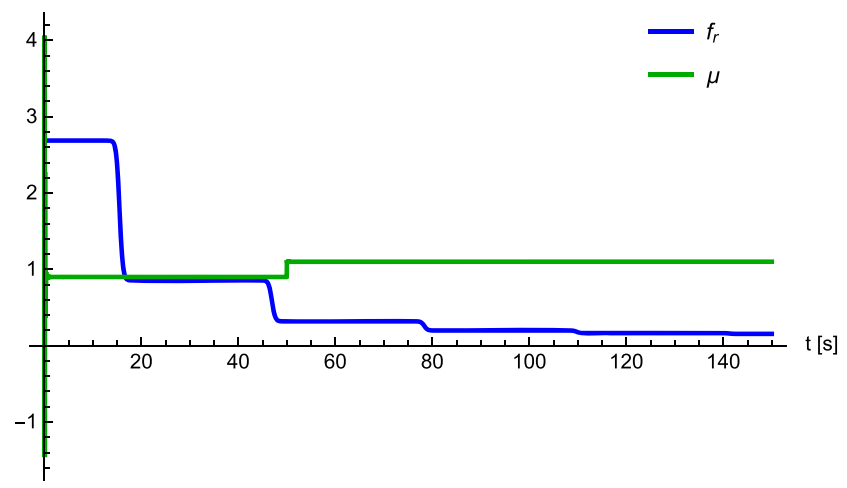

Fig. 6 Convergence of unknown parameters $f_{r}$ and $\mu$ in time

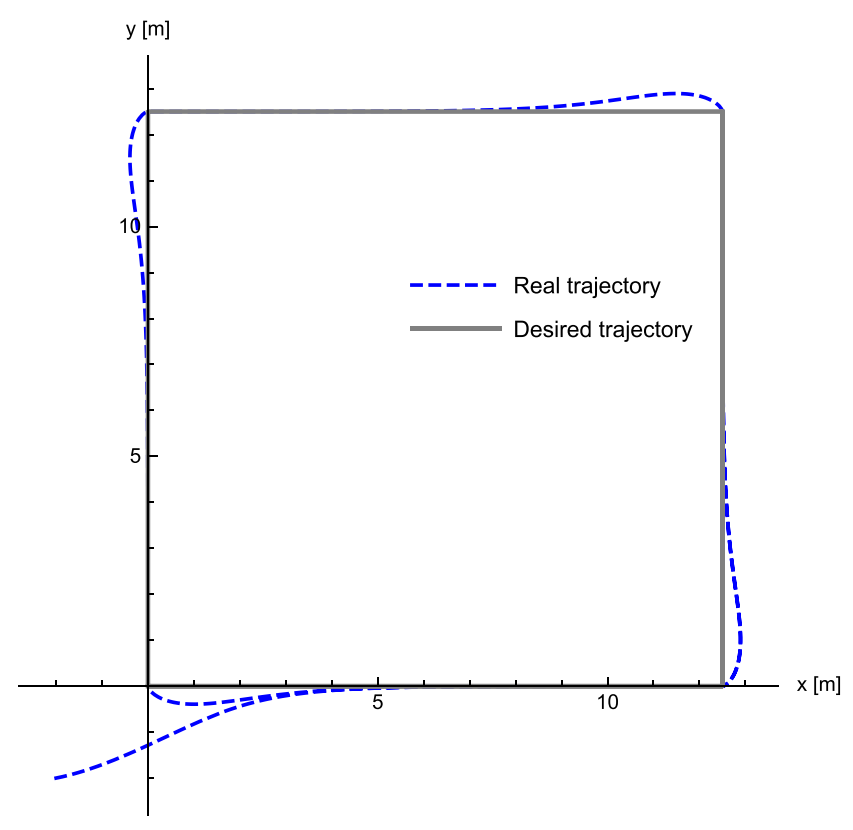

Fig. 7 Real and desired trajectory of the mobile platform

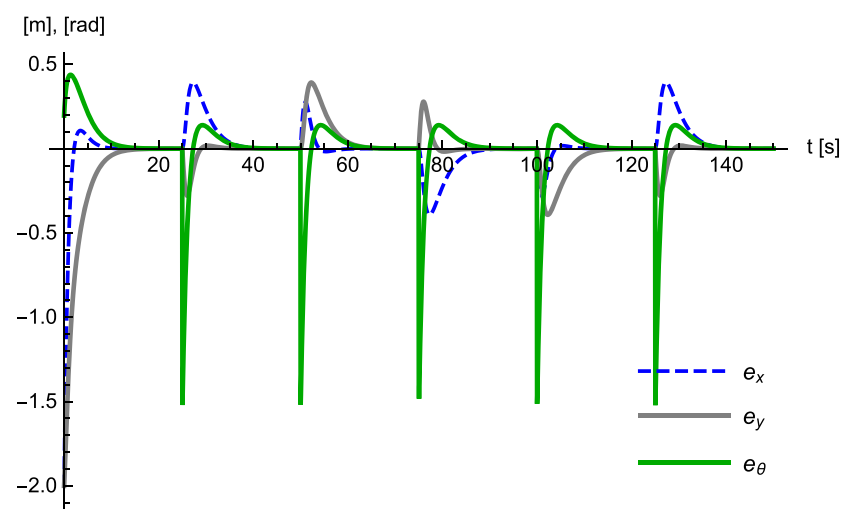

Fig. 8 Errors in Cartesian space - position and orientation of the mobile platform

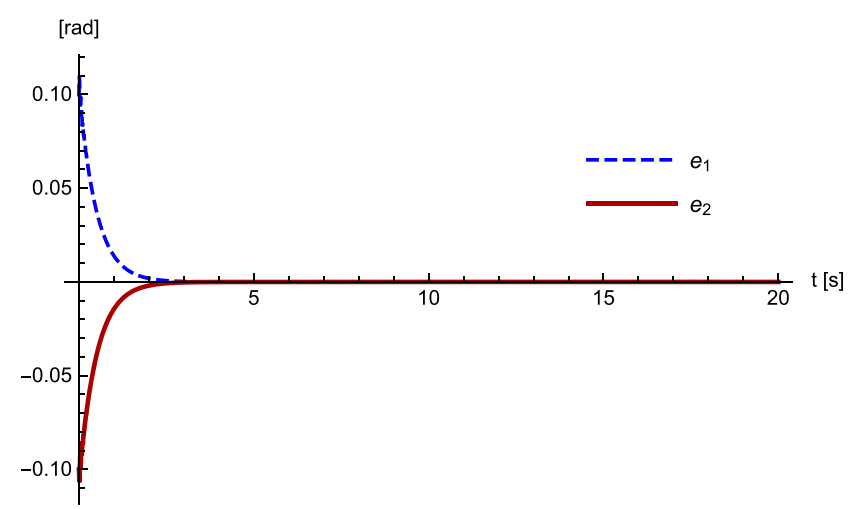

Fig. 9 Error in the manipulator's joint $q_{r 1}$ and $q_{r 2}$

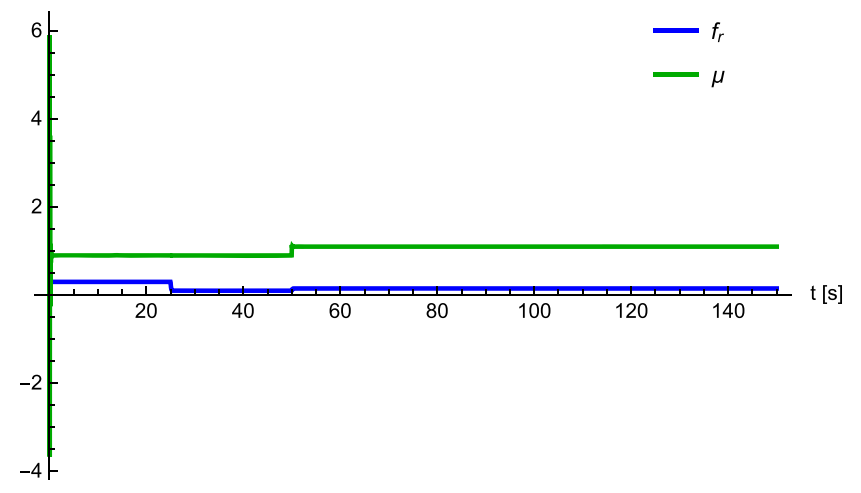

Fig. 10 Convergence of unknown parameters $f_{r}$ and $\mu$ in time 


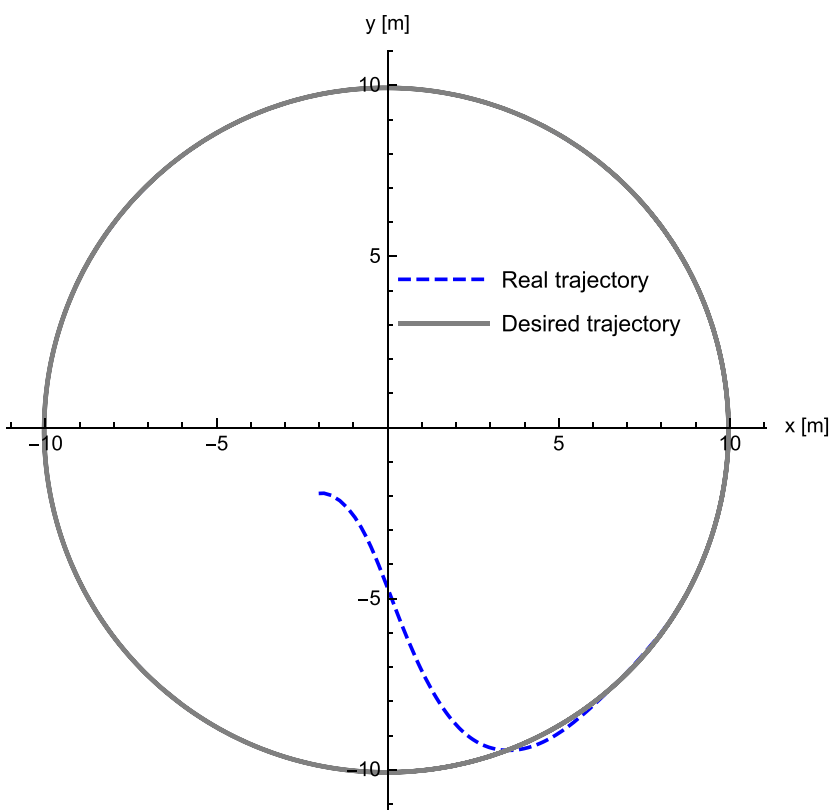

Fig. 11 Real and desired trajectory of the mobile platform

\subsection{Adaptive Version - An Admissible Trajectory}

A set of initial conditions for the experiment was assumed with following values: $x(0)=-2, y(0)=-2, \varphi(0)=\frac{\pi}{16}$, $q_{1}(0)=0.60, q_{2}(0)=-0.50, \hat{f}_{r}(0)=0.3, \hat{\mu}(0)=1.0$.

Dynamic controller coefficient $K_{d}=50$.

In Fig. 3 we can observe real and desired trajectory of the skid-steering platform. Tracking errors of the platform can be seen in Fig. 4.

In Fig. 5 trajectory tracking errors in manipulator joint space have been presented.

Figure 6 shows estimates of constant but unknown terrain parameters i.e. friction coefficients. It is easy to note that parameters do not converge to their real values.

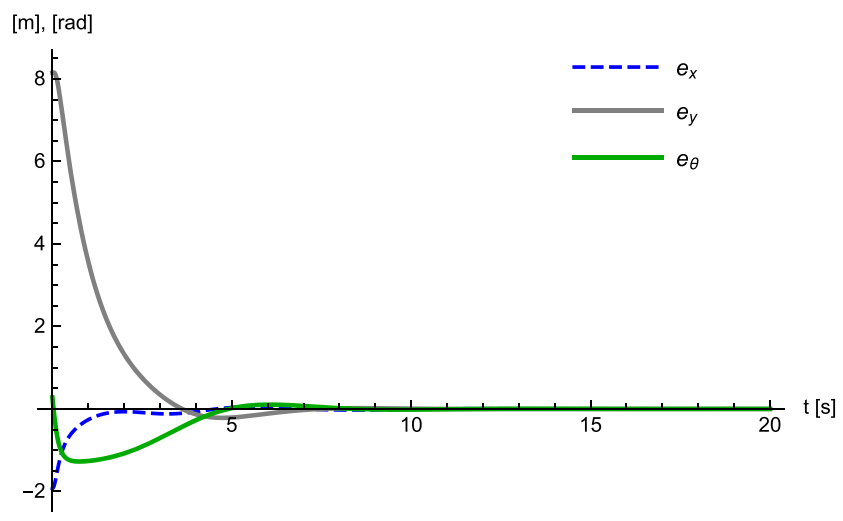

Fig. 12 Errors in Cartesian space - position and orientation of the mobile platform

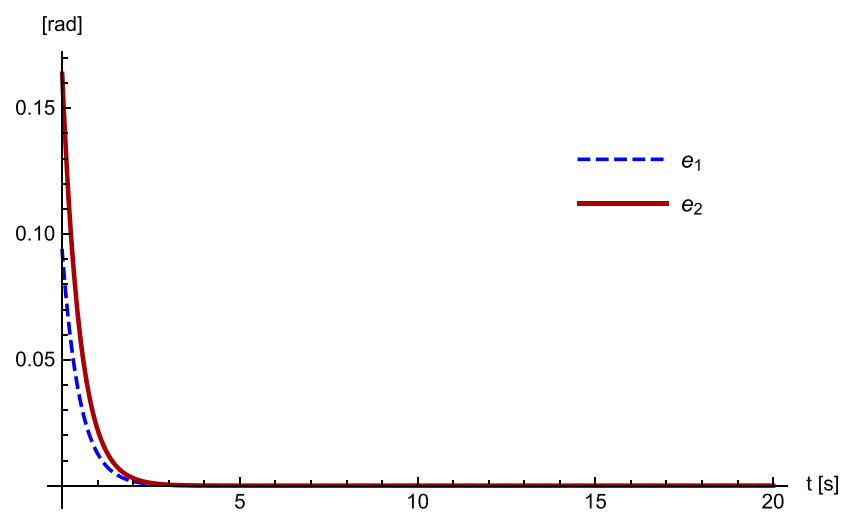

Fig. 13 Error in the manipulator's joint $q_{r 1}$ and $q_{r 2}$

\subsection{Adaptive Version - An Inadmissible Trajectory}

A set of initial conditions for the experiment was assumed with following values: $x(0)=-2, y(0)=-2, \varphi(0)=\frac{\pi}{16}$, $q_{1}(0)=0.60, q_{2}(0)=-0.50, \hat{f}_{r}(0)=0.3, \hat{\mu}(0)=1.0$.

Dynamic controller coefficient $K_{d}=50$.

In Fig. 7 we can observe real and desired trajectory of the skid-steering platform. Tracking errors of the platform can be seen in Fig. 8 .

In Fig. 9 trajectory tracking errors in manipulator joint space have been presented.

In Fig. 10 estimates of constant but unknown terrain parameters i.e. friction coefficients have been shown. It is easy to note that parameters do not converge to their real values.

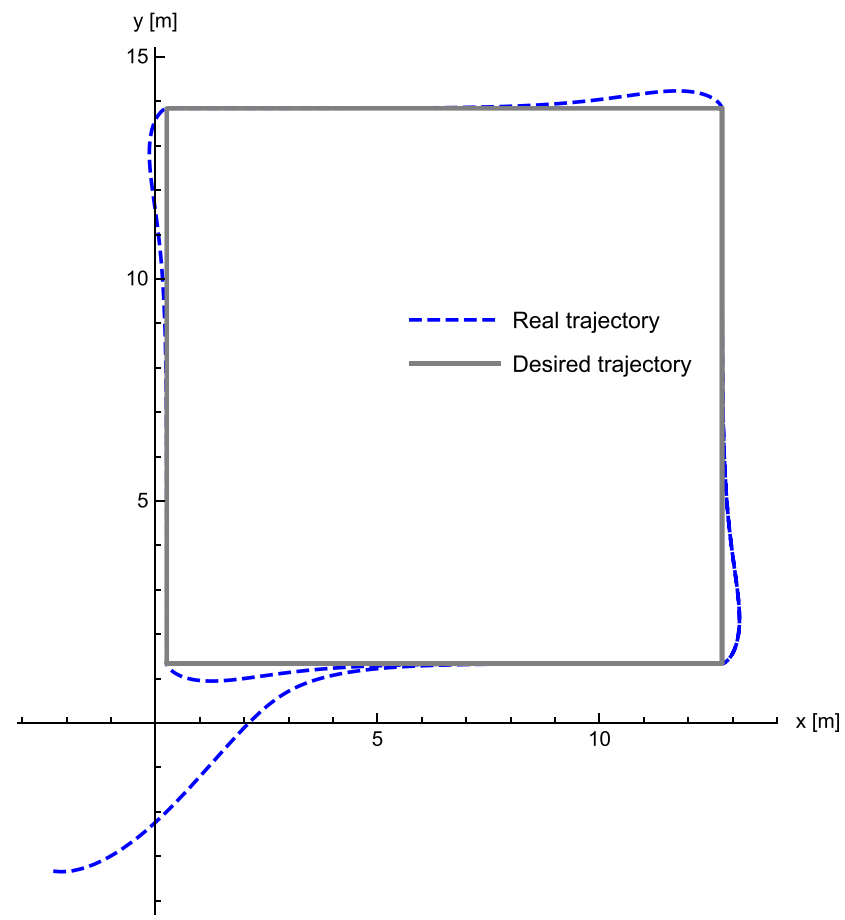

Fig. 14 Real and desired trajectory of the mobile platform 


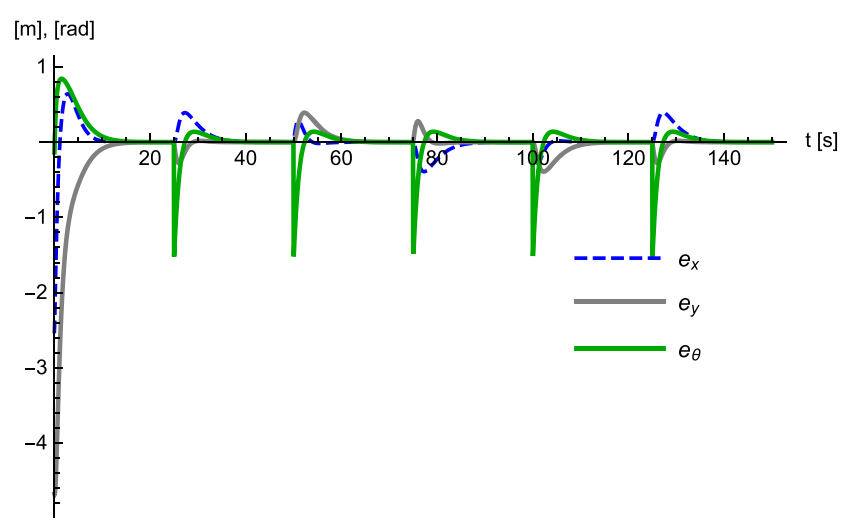

Fig. 15 Errors in Cartesian space - position and orientation of the mobile platform

\subsection{Robust Version - An Admissible Trajectory}

A set of initial conditions for the experiment was assumed with following values: $x(0)=-1.9, y(0)=-2, \varphi(0)=\frac{\pi}{9}$, $q_{1}(0)=0.58, q_{2}(0)=-0.21, f_{r}(0)=0.1, \mu(0)=0.1$.

Dynamic controller coefficients: $K_{d}=50$ and $K_{r}=50$.

In Fig. 11 we can observe real and desired trajectory of the skid-steering platform. Tracking errors of the platform can be seen in Fig. 12.

In Fig. 13 trajectory tracking errors in manipulator joint space have been presented.

\subsection{Robust Version - An Inadmissible Trajectory}

A set of initial conditions for the experiment was assumed with following values: $x(0)=-7, y(0)=-4.5, \varphi(0)=$ $-\frac{\pi}{9}, q_{1}(0)=0.51, q_{2}(0)=-0.88, f_{r}(0)=0.1, \mu(0)=$ 0.1 .

Dynamic controller coefficients: $K_{d}=50$ and $K_{r}=$ 150.

In Fig. 14 we can observe real and desired trajectory of the skid-steering platform. Tracking errors of the platform can be seen in Fig. 15.

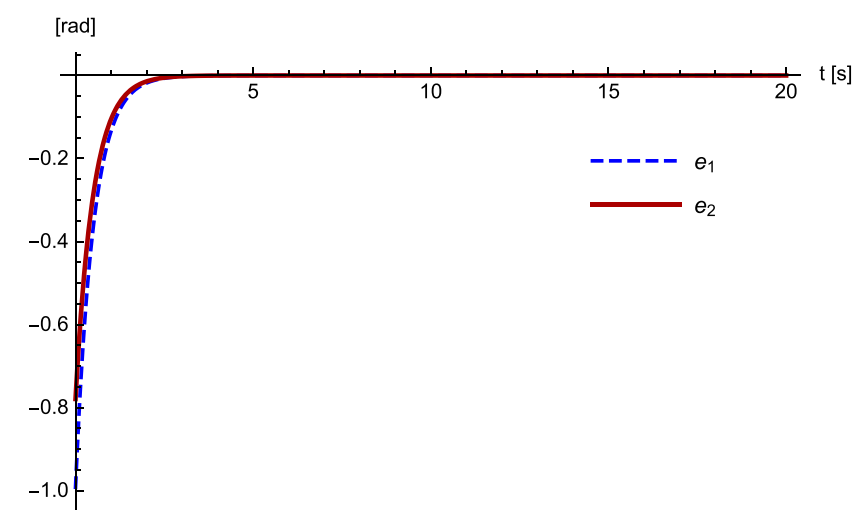

Fig. 16 Error in the manipulator's joint $q_{r 1}$ and $q_{r 2}$
In Fig. 16 trajectory tracking errors in manipulator joint space have been presented.

\section{Conclusions}

We have presented a concept of extended factitious force in regard to the control of mobile manipulators. A mobile manipulator structured of a skid-steering mobile platform and a rigid $2 \mathrm{R}$ manipulator was investigated as a research object. In many previous works we have presented the factitious force method and its extended version but in this article we assumed that there are some uncertainties of the environment the robot is moving in. To overcome those uncertainties we have proposed adaptive version of utilized dynamic control algorithm. Friction coefficients are constantly estimated and based on their estimation control signal is calculated to adapt to changing environment. We have also used robust version of the algorithm where we assume that there is a constant non-zero friction between wheels and the ground and based on the simulation results we have obtained it is clear that the algorithm is performing well. Furthermore, in both presented cases we assumed that the friction coefficients of environment surface are piecewise constant and despite this fact presented two approaches have good performance. Moreover, for each algorithm two scenarios were investigated. The first scenario was a desired trajectory of a circle - the admissible trajectory and the second scenario was a square - the inadmissible trajectory.

Acknowledgments The work presented here was supported by grant 0402/0069/17 for Wojciech Domski, by statutory grant 0401/0142/17 for Alicja Mazur and by grant 0402/0152/17 for Mirela Kaczmarek.

Open Access This article is distributed under the terms of the Creative Commons Attribution 4.0 International License (http:// creativecommons.org/licenses/by/4.0/), which permits unrestricted use, distribution, and reproduction in any medium, provided you give appropriate credit to the original author(s) and the source, provide a link to the Creative Commons license, and indicate if changes were made.

Publisher's Note Springer Nature remains neutral with regard to jurisdictional claims in published maps and institutional affiliations.

\section{References}

1. Frontline Robotics, Autonomous Perimeter Security. http://www. frontline-robotics.com/

2. Caracciolo, L., de Luca, A., Iannitti, S.: Trajectory tracking control of a four-wheel differentially driven mobile robot. In: Proceedings 1999 IEEE International Conference on Robotics and Automation (Cat. No.99CH36288C), vol. 4, pp. 2632-2638 (1999)

3. D'Andrea-Novel, B., Campion, G., Bastin, G.: Control of wheeled mobile robots not satisfying ideal velocity constraints: a singular 
perturbation approach. Int. J. Robust. Nonlinear Control 5(4), 243-267 (1995)

4. Domski, W., Mazur, A., Kaczmarek, M., Cholewiński, M.: Extended factitious force method in control of mobile manipulators with skid steering mobile platforms. In: Proceedings of the Polish National Conference on Robotics. Kudowa Zdrój, vol. 2, pp. 519-530. In Polish (2016)

5. Dulęba, I.: Modeling and Control of mobile manipulators. In: Proceedings of the 6th IFAC Symposium on Robot Control SYROCO'00, Vienna, pp. 687-692 (2000)

6. Gao, W., Jiang, Z.P.: Learning-based adaptive optimal tracking control of strict-feedback nonlinear systems. IEEE Trans. Neural Netw. Learn. Syst. 29(6), 2614-2624 (2018)

7. Kozłowski, K., Pazderski, D.: Modeling and control of a 4-wheel skid-steering mobile robot. Int. J. Appl. Math. Comput. Sci. 14(4), 4 (2004)

8. Krstić, M., Kanellakopoulos, I., Kokotović, P.: Nonlinear and Adaptive Control Design. Wiley, New York (1995)

9. Maalouf, E., Saad, M., Saliah, H.: A higher level path tracking controller for a four-wheel differentially steered mobile robot. Robot. Auton. Syst. 1(1), 23-33 (2006)

10. Mazur, A.: New approach to designing input-output decoupling controllers for mobile manipulators. Bull. Pol. Academy of Sci. Tech. 53(1), 31-37 (2005)

11. Mazur, A., Cholewiński, M.: Virtual force concept in steering mobile manipulators with skid-steering platform moving in unknown environment. J. Intell. Robot. Syst. 77, 433-443 (2015)

12. Mazur, A., Domski, W., Roszkowska, E.: Control of underactuated skid steering mobile platforms based on extended factitious force concept. J. Intell. Robot. Syst. 85, 1-19 (2016)

13. Canudas de Wit, C., Siciliano, B., Bastin, G.: Theory of Robot Control. Springer-Verlag, London (1996)

14. Mohammadpour, E., Naraghi, M., Gudarzi, M.: Posture stabilization of skid steer wheeled mobile robots. In: Proceedings of the IEEE International Conference on Robotics Automation and Mechatronics, Singapore, pp. 163-169 (2010)

15. Motte, I., Campion, G.: A slow manifold approach for the control of mobile robots not satisfying the kinematic constraints. IEEE Trans. Robot. Autom. 16(6), 875-880 (2000)

16. Pazderski, D., Kozłowski, K.: Trajectory tracking of underactuated skid-steering robot. In: Proceedings of the American Control Conference, Seattle, pp. 3506-3510 (2008)

17. Samson, C., Ait-Abderrahim, K.: Feedback control of a nonholonomic wheeled cart in cartesian space. In: Proceedings of the IEEE International Conference on Robotics and Automation, Sacramento, pp. 1136-1141 (1991)

18. Slotine, J.J.E., Li, W.: Adaptive manipulator control: a case study. IEEE Trans. Autom. Contr. 33(11), 995-1003 (1988)

19. Tchoń, K., Mazur, A., Dulęba, I., Hossa, R., Muszyński, R.: Mobile Manipulators and Robots: Models, Motion Planning, Control. PLJ Publisher, Warsaw (2000). (in Polish)
20. Yamamoto, Y., Yun, X.: Effect of the dynamic interaction on coordinated control of mobile manipulators. IEEE Trans. Robot. Autom. 12(5), 816-824 (1996)

21. Zadarnowska, K., Ratajczak, A.: Task-priority motion planning of wheeled mobile robots subject to slipping. Lecture Notes Control Inform. Sci. 422, 75-85 (2012)

Wojciech Domski in 2013 received his bachelor degree in Control Engineering and Robotics at Wrocław University of Science and Technology. In 2014 he successfully continued his education and received master degree in Control Engineering and Robotics at Wrocław University of Science and Technology. Also in 2014 he received Dean's award for the best students of the faculty of Electronics and later that year he received first prize in TOP10 competition for the best graduate at WUST on the faculty level. From 2013 to 2017 he worked in ReMeDi ("Remote Medical Diagnostician") EU FP7 project. Since 2015 he works at Chair of Cybernetics and Robotics as a research assistant. In 2017 he also received his second bachelor degree in Computer Science at WUST which gave him first prize in TOP10 competition for the best graduate at Wrocław University of Science and Technology. He is currently a $\mathrm{Ph}$.D. student at WUST where his main research area are extended factitious force, mobile manipulators and space robotics.

Alicja Mazur received the M.Sc. degree in automatics and the Ph.D. degree in robotics from the Wrocław University of Technology, Wrocław, Poland, in 1990 and 1996, respectively. In 1997 she was a visiting professor at the Institute for Robotics and Process Control in Brunswick, Germany. In 2011 she received D. Sc. degree in robotics from Lodz University of Technology. From 2015 she is chief of Chair of Cybernetics and Robotics, Faculty of Electronics at Wrocław University of Science and Technology. Her current research interests include the dynamics and control of robotic systems, nonlinear control theory of nonholonomic systems, geometric control theory, universal and adaptive control, mobile robotics, space robots.

Mirela Kaczmarek received her MSc degree in Control Engineering and Robotics from Wrocław University of Science and Technology, Poland, in 2012. During the studies she gained an internship at LIREC ("Living with Robots and Interactive Companion") EU FP7 project. She is currently a Ph.D. student at Chair of Cybernetics and Robotics of WUST. Her research interests cover position-force control algorithms for stationary and mobile manipulators, as well as social robotics. She is an author of 12 publications. 